Analysis and wafer-level design of a high-order silicon vibration isolator for resonating MEMS devices

This article has been downloaded from IOPscience. Please scroll down to see the full text article.

2011 J. Micromech. Microeng. 21015017

(http://iopscience.iop.org/0960-1317/21/1/015017)

View the table of contents for this issue, or go to the journal homepage for more

Download details:

IP Address: 141.211.173.82

The article was downloaded on 06/04/2012 at 16:58

Please note that terms and conditions apply. 


\title{
Analysis and wafer-level design of a high-order silicon vibration isolator for resonating MEMS devices
}

\author{
Sang Won Yoon ${ }^{1,2}$, Sangwoo Lee ${ }^{1}$, Noel C Perkins ${ }^{3}$ and Khalil Najafi ${ }^{1}$ \\ ${ }^{1}$ Department of Electrical Engineering and Computer Science, University of Michigan, \\ Ann Arbor, MI, USA \\ 2 Toyota Research Institute of North America, Ann Arbor, MI, USA \\ ${ }^{3}$ Department of Mechanical Engineering, University of Michigan, Ann Arbor, MI, USA
}

Received 8 August 2010, in final form 26 November 2010

Published 22 December 2010

Online at stacks.iop.org/JMM/21/015017

\begin{abstract}
This paper presents the analysis and preliminary design, fabrication, and measurement for mechanical vibration-isolation platforms especially designed for resonating MEMS devices including gyroscopes. Important parameters for designing isolation platforms are specified and the first platform (in designs with cascaded multiple platforms) is crucial for improving vibration-isolation performance and minimizing side-effects on integrated gyroscopes. This isolation platform, made from a thick silicon wafer substrate for an environment-resistant MEMS package, incorporates the functionalities of a previous design including vacuum packaging and thermal resistance with no additional resources. This platform consists of platform mass, isolation beams, vertical feedthroughs, and bonding pads. Two isolation platform designs follow from two isolation beam designs: lateral clamped-clamped beams and vertical torsion beams. The beams function simultaneously as mechanical springs and electrical interconnects. The vibration-isolation platform can yield a multi-dimensional, high-order mechanical

low pass filter. The isolation platform possesses eight interconnects within a $12.2 \times 12.2 \mathrm{~mm}^{2}$ footprint. The contact resistance ranges from 4-11 $\Omega$ depending on the beam design. Vibration measurements using a laser-Doppler vibrometer demonstrate that the lateral vibration-isolation platform suppresses external vibration having frequencies exceeding $2.1 \mathrm{kHz}$.
\end{abstract}

(Some figures in this article are in colour only in the electronic version)

\section{Introduction}

Vibration has profound impact on the performance of MEMS devices and is a well-recognized source of output errors. Since many MEMS devices sense the dynamic displacement of device structures, the undesired vibration-induced displacements of these structures result in unpredictable/false outputs that also are difficult to compensate with electronics. The errors can be particularly significant for high $Q$ resonant devices such as gyroscopes, because low damping may amplify vibration in critical frequency bands [1-3].

Common remedies are to incorporate macro-scale massspring-damper systems or simple dampers [4]. However, such chip-level solutions typically increase device size and cost. These limitations can be resolved by integrating MEMS devices with micromachined mass-spring-damper structures [5-8]. The micromachined structures serve as passive, mechanical low pass filters (LPF) and essentially attenuate high frequency environmental vibration. The performance of the passive LPF can be further improved by employing active vibration isolators $[9,10]$, which however require more complex structures, are harder to fabricate, consume additional power, and increase device size [11].

An alternative solution for improving the vibration performance of a LPF is the integration or cascading of multiple vibration-isolation platforms. The multiple platforms form a high-order LPF and greatly reduce vibration amplitude. However, this solution may also increase device size and fabrication complexity, and may also degrade device 


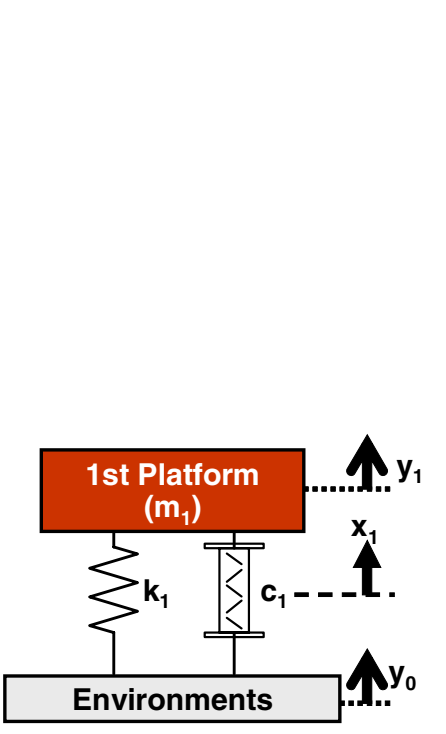

(a)

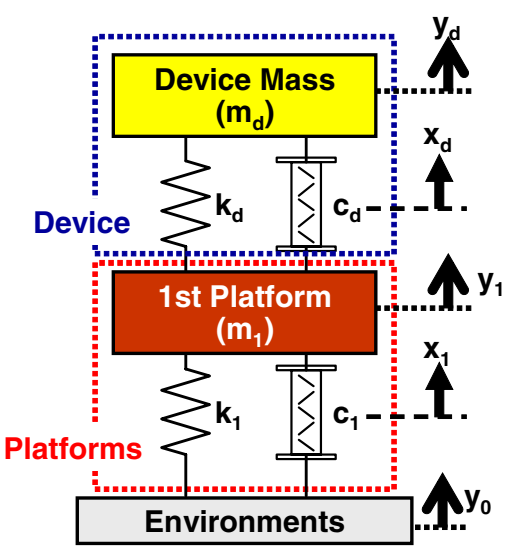

(b)

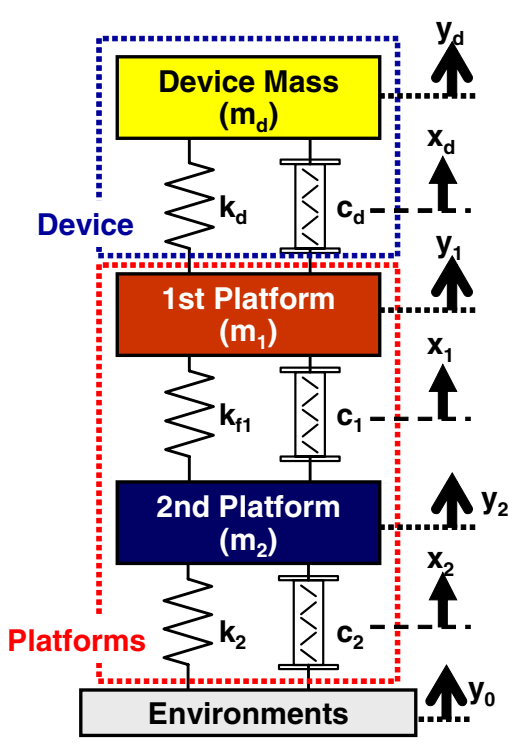

(c)

Figure 1. Schematics and models of $(a)$ a vibration-isolation platform without an integrated device, $(b)$ device integrated with one-stage vibration-isolation platforms, $(c)$ device integrated with two-stage vibration-isolation platforms.

performance, including the shifting of resonant frequencies or reducing the $Q$ of integrated gyroscopes [5, 12]. Therefore, the need remains for a simple approach that integrates micromachined vibration isolators at the wafer level without degrading gyroscope performance. In addition, many reported micromachined vibration isolators are not vacuum packaged [6-10]; thus, their $Q$ are expected to be substantially smaller than the $Q$ of vacuum packaged MEMS gyroscopes. Thus, the effect of the isolator $Q$ on gyroscope performance requires clarification.

We previously reported an environment-resistant packaging technology [13] that provides thermal and vibration isolation to vacuum packaged MEMS devices via a glass platform with mounting springs. Due to limitations of glass micromachining, we demonstrated vibration isolation solely along the vertical direction.

This paper presents a wafer-level approach to integrate lateral and vertical vibration-isolation platforms with our previous environment-resistant package. Two platform designs are proposed that enable one to integrate the platform along the most vibration-sensitive direction of the integrated gyroscopes. It is reported that many MEMS gyroscopes are susceptible to vibration along the sensing axis (i.e. the direction of Coriolis force) [3]. Our concept can realize a multi-dimensional filtering structure with lateral and vertical designs simultaneously fabricated via a single fabrication process. This goal is achieved by employing high-aspect-ratio springs fabricated on thick single crystal silicon. Theoretical analyses and simulation results are also presented to (1) estimate the performance of the MEMS devices with integrated vibration-isolation platforms, and (2) understand and reduce potential drawbacks. The design concept can also yield a multi-axis, high-order mechanical LPF for superior vibration isolation. The feasibility of this concept is demonstrated herein through proof-of-concept fabrication and vibrationperformance testing.

\section{Theory and analysis}

\subsection{Model definition}

Figure 1 provides the schematics of the vibration-isolation platform(s) integrated with or without a device. The principal variables illustrated therein are as follows. The quantities $x_{d}, x_{1}, x_{2}$ represent the stretch of the device spring, the first platform spring, and the second platform spring, respectively. The quantities $y_{d}, y_{1}, y_{2}$ denote the absolute displacements of the device mass, the first platform mass, and the second platform mass, respectively. The absolute displacement of the substrate (i.e. the vibration excitation) is denoted by $y_{0}$ and $\left(m_{d}, c_{d}, k_{d}\right),\left(m_{1}, c_{1}, k_{1}\right)$, and $\left(m_{2}, c_{2}, k_{2}\right)$ denote the mass, damping, and stiffness of the device, first platform, and second platform, respectively.

For the design shown in figure 1( $a$ ), defining a platform not integrated with a device, the transmissibility, under assumed harmonic excitation, is given by [11]

$$
\left|\frac{Y_{1}}{Y_{0}}\right|=\left[\frac{1+(2 \zeta r)^{2}}{\left(1-r^{2}\right)^{2}+(2 \zeta r)^{2}}\right]^{1 / 2}
$$

where $r=\omega / \omega_{1}$ and $\zeta=c_{1} /\left(2^{*} m_{1}{ }^{*} \omega_{1}\right)$. Here, $Y_{0}$ and $Y_{1}$ are the amplitudes of $y_{0}$ and $y_{1}, r$ is the ratio of the excitation frequency to the natural frequency, and $\zeta$ is the damping ratio [14].

For the design shown in figure $1(b)$, defining a platform integrated with a device, the equations of motion are given by Device : $m_{d}\left(\ddot{x}_{d}+\ddot{x}_{1}+\ddot{x}_{0}\right)=-k_{d} x_{d}-c_{d} \dot{x}_{d}$,

First platform : $\quad m_{1}\left(\ddot{x}_{1}+\ddot{x}_{0}\right)=k_{d} x_{d}+c_{d} \dot{x}_{d}-k_{1} x_{1}-c_{1} \dot{x}_{1}$. 


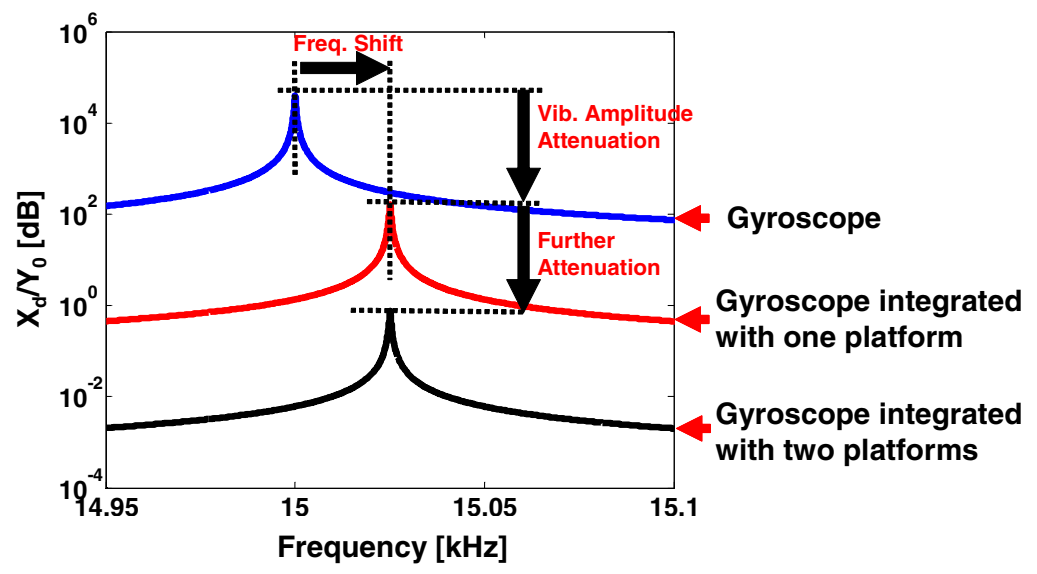

(a)

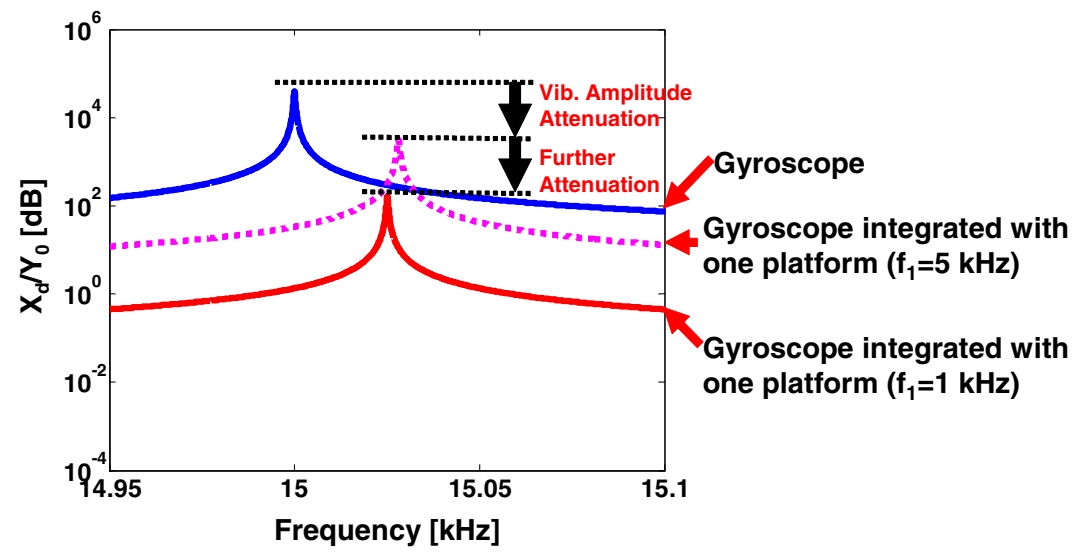

(b)

Figure 2. The frequency response of an example MEMS gyroscope with integrated vibration-isolation platform(s). (a) Comparison of one versus two platforms. $(b)$ One platform with two different platform resonant frequencies $\left(f_{1}\right)$.

Taking the Laplace transform of (2) and (3) yields

$$
\begin{gathered}
{\left[\begin{array}{c}
X_{d} / X_{0} \\
X_{1} / X_{0}
\end{array}\right]=\left[\begin{array}{cc}
m_{d} s^{2}+c_{d} s+k & m_{d} s^{2} \\
-c_{d} s-k_{d} & m_{1} s^{2}+c_{1} s+k_{1}
\end{array}\right]^{-1}} \\
\cdot\left[\begin{array}{l}
-m_{d} s^{2} \\
-m_{1} s^{2}
\end{array}\right]
\end{gathered}
$$

from which

$\frac{X_{d}}{X_{0}}=$

$\frac{-m_{d} c_{1} s^{3}-k_{1} m_{d} s^{2}}{m_{d} m_{1} s^{4}+\left(m_{d} c_{d}+m_{d} c_{1}+m_{1} c_{d}\right) s^{3}+\left(m_{d} k_{d}+m_{d} k_{1}+m_{1} k_{d}+c_{d} c_{1}\right) s^{2}+\left(c_{d} k_{1}+c_{1} k_{d}\right) s+k_{d} k_{1}}$.

Here $X_{d}, X_{1}$ and $X_{o}$ denote the Laplace transforms of $x_{d}, x_{1}$ and $x_{o}$, respectively, and $s=\mathrm{j} \omega$.

For the design shown in figure 1(c), defining two-stage vibration-isolation platforms integrated with a device, the equations of motion are given by

Device : $m_{d}\left(\ddot{x}_{d}+\ddot{x}_{1}+\ddot{x}_{2}+\ddot{x}_{0}\right)=-k_{d} x_{d}-c_{d} \dot{x}_{d}$,

First platform : $m_{1}\left(\ddot{x}_{1}+\ddot{x}_{2}+\ddot{x}_{0},\right)$

$$
=k_{d} x_{d}+c_{d} \dot{x}_{d}-k_{1} x_{1}-c_{1} \dot{x}_{1} \text {, }
$$

Second platform: $m_{2}\left(\ddot{x}_{2}+\ddot{x}_{0}\right)=k_{1} x_{1}+c_{1} \dot{x}_{1}-k_{2} x_{2}-c_{2} \dot{x}_{2}$.
Following the analogous steps above, we arrive at

$$
\begin{aligned}
& {\left[\begin{array}{l}
X_{d} / X_{0} \\
X_{1} / X_{0} \\
X_{2} / X_{0}
\end{array}\right]} \\
& =\left[\begin{array}{ccc}
m_{d} s^{2}+c_{d} s+k_{d} & m_{d} s^{2} & m_{d} s^{2} \\
-c_{d} s-k_{d} & m_{1} s^{2}+c_{1} s+k_{1} & m_{1} s^{2} \\
0 & -c_{1} s-k_{1} & m_{2} s^{2}+c_{2} s+k_{2}
\end{array}\right] \\
& \qquad\left[\begin{array}{c}
-m_{d} s^{2} \\
-m_{1} s^{2} \\
-m_{2} s^{2}
\end{array}\right] .
\end{aligned}
$$

Figure 2(a) compares the frequency responses computed for an example MEMS gyroscope [15] integrated with one versus two vibration-isolation platforms (refer to figures $1(b)$, (c)). The example gyroscope has a resonant frequency of $15 \mathrm{kHz}$ and a high $Q$ of $\sim 40 \mathrm{k}$ (measured in vacuum). The results clearly demonstrate that the frequency response of the integrated gyroscope is substantially attenuated by the vibration-isolation platform(s) and that the attenuation is greater for the two-stage platform (representing a higherorder mechanical LPF). Moreover, as illustrated in figure $2(b)$, the attenuation depends on the resonant frequency of the integrated platform, as expected. Therefore, there is a significant advantage in using cascaded, multiple vibrationisolation platforms. However, these results also reveal that 


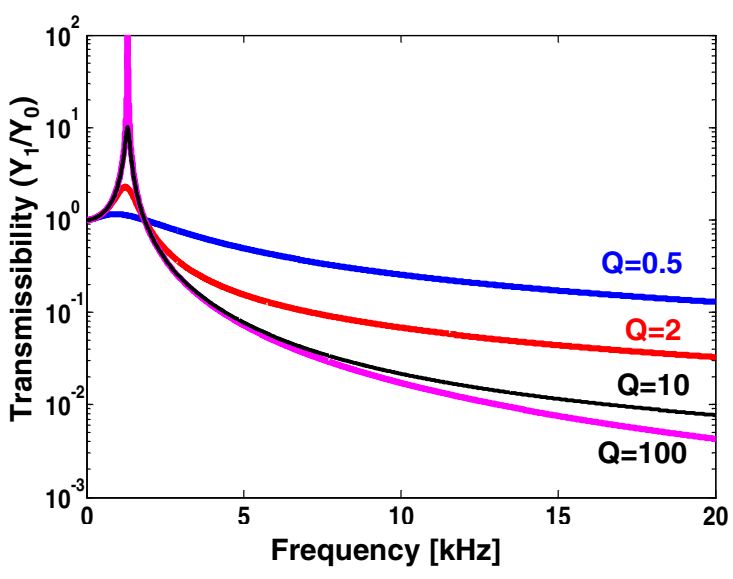

(a)

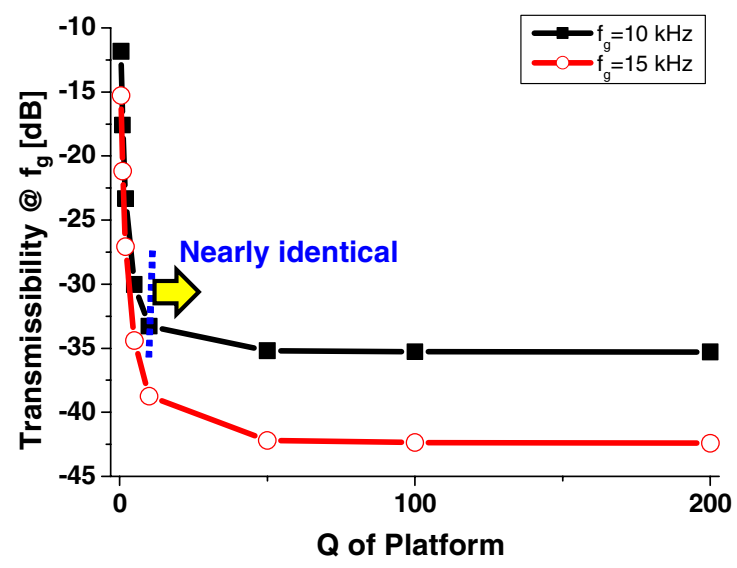

(b)

Figure 3. Transmissibility of a vibration-isolation platform (figure $1(a)$ ) for various values of the platform $Q$. (b) Transmissibility at typical gyro resonant frequencies $\left(f_{g}=10\right.$ or $\left.15 \mathrm{kHz}\right)$.

the platform(s) integration alters the resonant frequency of the gyroscope, which may also compromise gyro performance. This unwanted effect can be minimized using the methods discussed next.

\subsection{Design rules for vibration-isolation platforms for MEMS gyroscopes}

The objective in designing for vibration isolation is to minimize the vibration transmissibility [14] while also minimizing possible side-effects. The transmissibility quantitatively measures how much vibration is transmitted to the device through the vibration-isolation platform, and thus, it measures the degree of vibration isolation. The integration of a vibration-isolation platform may also result in adverse sideeffects. As figure 2 depicts, adding the platform may change the resonance frequency of the device and this change may degrade device performance. Similarly, the added platform may also decrease the device $Q$ which again may degrade performance. Both the resonant frequency and $Q$ are important for gyroscope performance.

Figure 3 illustrates the transmissibility of a platform shown in figure $1(a)$ for various $Q$. Figure $3(b)$ shows the transmissibility at two specific frequencies (10 and $15 \mathrm{kHz}$ ), which are target values for the resonant frequency of MEMS gyroscopes [15, 16]. Note that while high $Q$ reduces the transmissibility at these specific frequencies (i.e. better vibration isolation results), this improvement quickly diminishes for $Q$ exceeding a threshold value (e.g. approximately $Q=10$ for $f_{g}=10 \mathrm{kHz}$ ). Therefore, the $Q$ of the vibration-isolation platform exceeding the threshold yields negligible improvement in vibration isolation at the target (high) frequencies and may ultimately degrade the performance of integrated devices at/near the (lower) resonant frequency of the platform; refer to figure $3(a)$.

Two example gyroscopes are selected to investigate their performance change when integrated with one vibrationisolation platform (figure $1(b)$ ). One gyroscope has relatively high $Q(\sim 40 \mathrm{k})$ and high resonant frequency $(15 \mathrm{kHz})$
[15], and the other has relatively low $Q(\sim 4.1 \mathrm{k})$ and low resonant frequency $(8.9 \mathrm{kHz})[16,17]$. Figure 4 illustrates the performance change of the gyroscopes integrated with one vibration-isolation platform.

We briefly summarize salient findings here that are pertinent to the results of this study using analyses from this paper or our previous work [12].

First, the two most important parameters are the resonant frequency and the mass of the 'first' platform. When multiple vibration-isolation platforms are used, the first platform refers to the platform directly connected to the device, as illustrated in figure $1(b)$. In a single-platform design, the first platform is the device platform itself. The transmissibility decreases by reducing the resonant frequency of the first platform, as depicted in figure $2(b)$. Moreover, minimal change to the resonant frequency and minimal reduction in $Q$ follows from increasing the mass of the first platform.

Second, the integration of two (or multiple) platforms further reduces vibration transmission because they form highorder LPF, as shown in figure 2(a). Specially, an $N$-platform design forms a $2 N$ th-order LPF, whereas a single-platform design forms a second-order filter. The transmissibility reduction is nearly independent of the masses of the first and second platforms.

Third, the $Q$ of the first platform influences the performance of the integrated gyroscopes and the vibrationisolation platform, but to a lesser extent when compared to the mass of the first platform. High platform $Q$ decreases the transmissibility at the gyro resonant frequency, but this effect becomes minor for $Q$ exceeding a threshold, as explained above and as shown in figure $3(b)$. The platform $Q$ shifts the resonant frequency of the integrated gyroscope by a negligible amount, but it strongly reduces the $Q$ of the gyroscope, as illustrated in figure 4. However, as also illustrated in figure 4 , this effect can be substantially mitigated by increasing the platform mass.

Fourth, the mass and stiffness characteristics of the second platforms are not as critical as those of the first platform. The resonant frequency and $Q$ are only weakly dependent on these characteristics. 


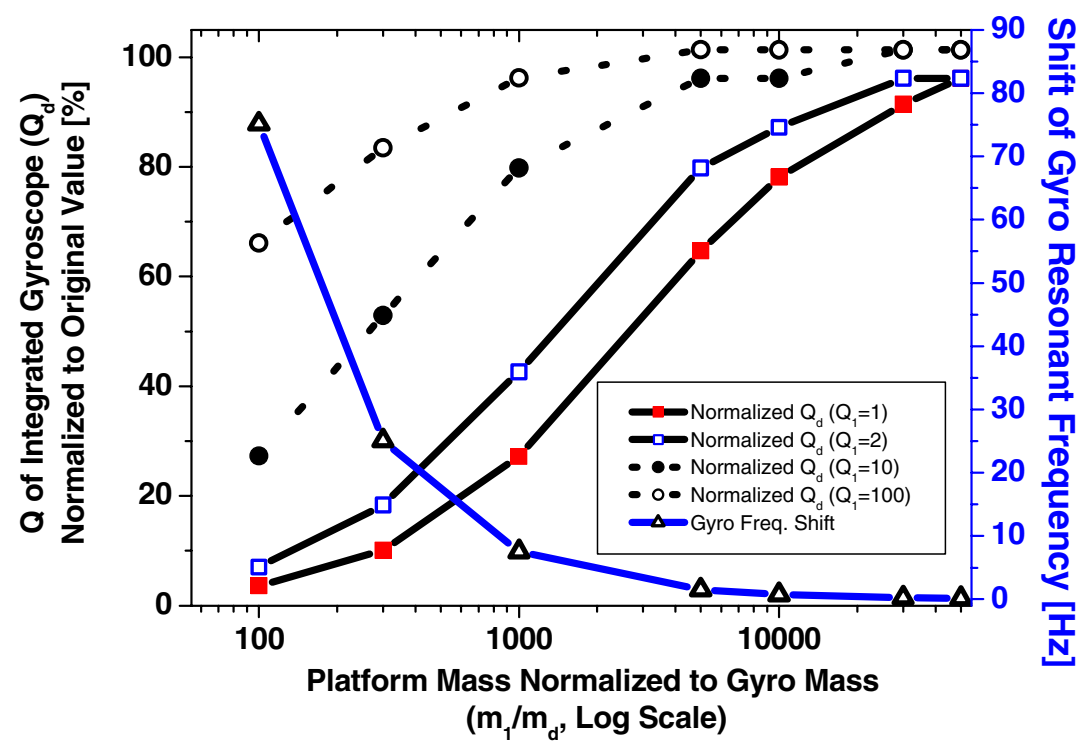

(a)

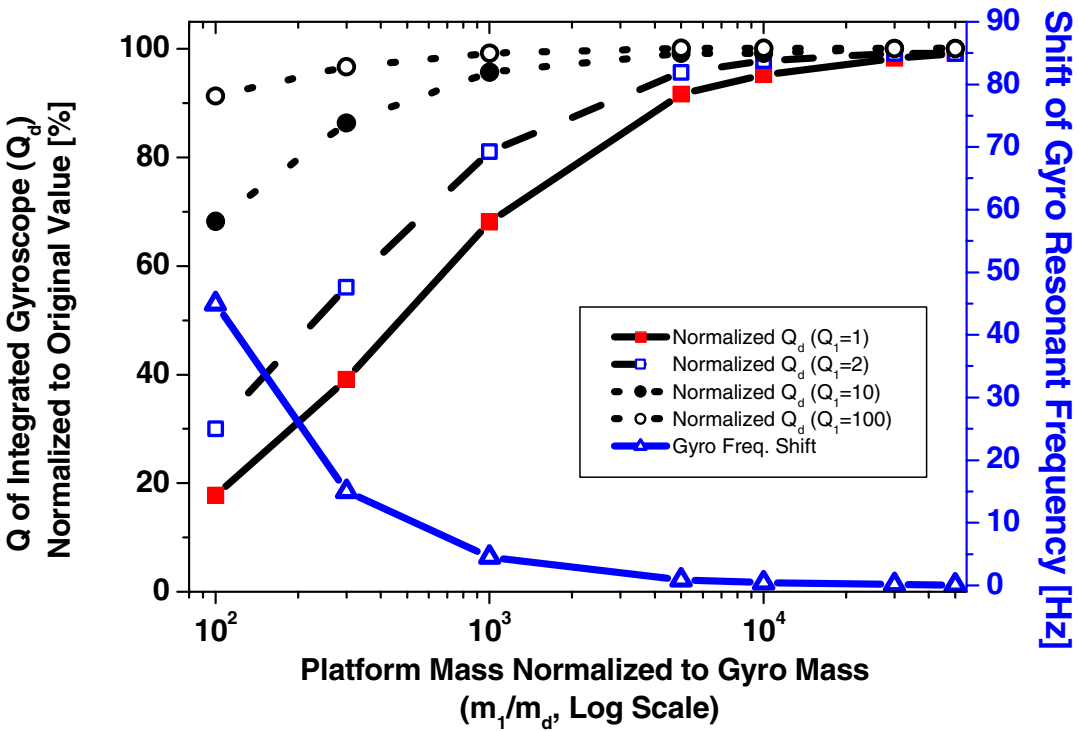

(b)

Figure 4. The $Q$ and resonant frequency of a gyroscope integrated with one vibration-isolation platform (figure $1(b))$ as functions of platform $Q\left(Q_{1}\right)$ and mass $\left(m_{1}\right)$. (a) Relatively high $Q$ gyroscope ( $Q$ of $\sim 40 \mathrm{k}$ ), (b) relatively low $Q$ gyroscope $(Q$ of $\sim 4.1 \mathrm{k})$.

Finally, the vibration-isolation platforms are the most efficient when aligned along the sense direction of gyroscopes $[3,12]$.

In summary, the design of the first platform is crucial whereas multiple platforms lead to higher-order filtering. It is also important to note that most of the drawbacks associated with integrating low $Q$ platform(s) can be resolved by simply employing larger platform masses.

\section{Concept and design}

\subsection{Concept}

Based on the above findings, we focus on the design of the first platform, with the goals of increasing the platform mass and decreasing the resonant frequency, and the development of a fabrication process to integrate multiple platforms for lateral or vertical motions.

Figure 5 depicts the schematic of the proposed vibrationisolation platform integrated with an example MEMS device, a SOG (silicon-on-glass) device in the figure. The platform consists of platform mass, isolation beams, vertical feedthroughs (VFTs), and bonding pads. In contrast to the previous approach [13], the isolation beams are located outside of the device package, and they connect the VFTs and the bonding pads on the substrate. Therefore, flip-chip bonded devices on our platform do not need any wire-bonding, and thus, this new design achieves true wafer-level packaging with built-in vibration isolation. In addition, this concept easily incorporates multiple cascaded isolation platforms, thereby realizing a high-order LPF to dramatically suppress vibration effects [12] as shown in figure 5(b). 


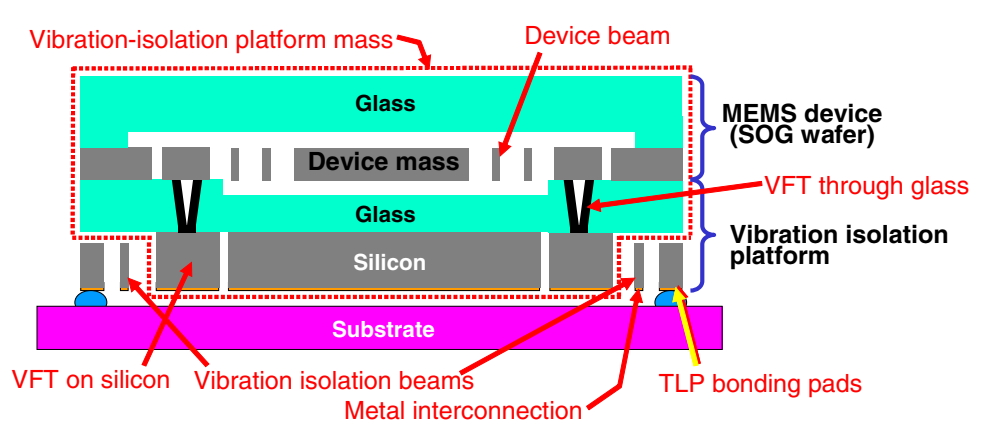

(a)

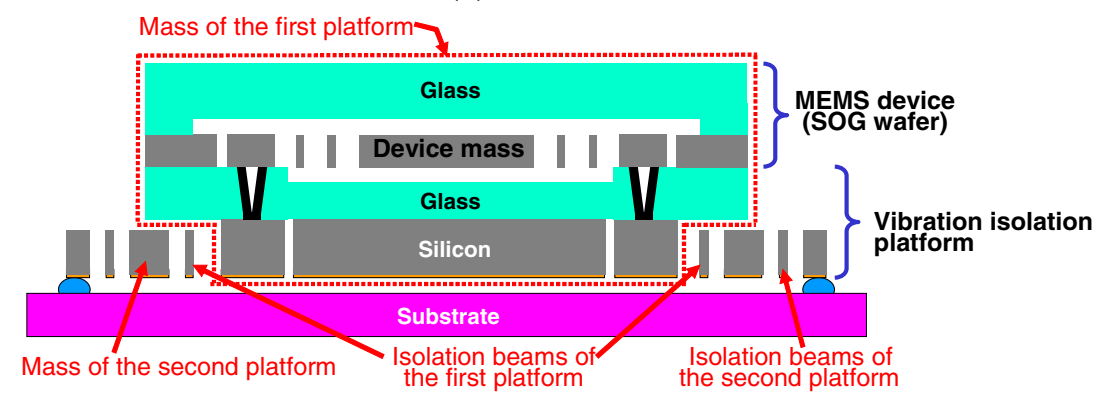

(b)

Figure 5. (a) Schematic of the proposed vibration isolator structure. A MEMS device wafer (upper part) is bonded to the vibration isolator wafer (lower part) at the wafer level. (b) Modified vibration isolator structure with multiple cascade platforms.

The isolation beams simultaneously serve as mechanical springs and electrical interconnects. Multiple isolation-beam designs may be required to optimize the performance for both lateral and vertical vibration isolation, and these can be simultaneously integrated on a single wafer using an identical fabrication process. The interconnect resistance remains low by employing beams fabricated from highly-doped single crystal silicon with additional deposited metal layers.

The platform mass remains much larger than the device mass so as to decrease the resonant frequency of the platform while minimizing performance changes of the integrated device, such as changes to its resonant frequency and/or $Q$ (refer to section 2) and without any additional wafers, material, or fabrication steps. As evident, the two example gyroscopes (described in section 2) experience negligible resonant frequency shift and minor $Q$ reduction when integrated with our lateral vibration-isolation platform that has a fundamental resonant frequency of $\sim 1 \mathrm{kHz}$ and measured $Q$ of $\sim 2.2$ (as will be presented in the following sections). The relatively high $Q$ gyroscope ( $Q$ of $\sim 40 \mathrm{k}$, resonant frequency of $15 \mathrm{kHz}$ ) [15] experiences a resonant frequency shift of only $\sim 0.75 \mathrm{~Hz}$ and a minor $Q$ reduction of $\sim 13 \%$. The relatively low $Q$ gyroscope ( $Q$ of $\sim 4.1 \mathrm{k}$, resonant frequency of $\sim 8.9 \mathrm{kHz})[16,17]$ experiences a resonant frequency shift of only $\sim 0.9 \mathrm{~Hz}$ and a minor $Q$ reduction of $\sim 4 \%$. This approach can also relieve packaging stresses induced from the mismatch of thermal expansion coefficients between the package and its substrate because the package is bonded to the substrate through compliant springs.

This approach is compatible with previously reported environment-resistant packages [13]. In doing so, one achieves an ideal concept for an environment-resistant package that provides multi-axis vibration isolation, thermal isolation, vacuum packaging, package stress relaxation, and (optional) vacuum encapsulation. Moreover, the vibration-isolation platform is fabricated using the silicon substrate of the previous package, and thus, it does not require additional resources (e.g. wafers) or significant increase in package size (area or vertical profile).

\subsection{Structure design}

We propose two integrated designs for the vibration-isolation platform, one for lateral and one for vertical vibration isolation. The views of both designs are depicted in figure 6 . The lateral design utilizes clamped-clamped beams as springs whereas the vertical design employs torsion beams as also demonstrated in previous studies $[18,19]$. For simplicity, the silicon mass surrounding the beams is not included in the figure or the following FEM simulations using ANSYS ${ }^{\mathrm{TM}}$. Each design incorporates eight vibration-isolation beams, which bridge VFTs and bonding pads.

Both the lateral and vertical designs are compatible with the previous environment-resistant package [13]. The VFTs conform to the footprints of the previous package, and the isolation beams are fabricated only at the perimeter of the silicon substrate to free up the center for vacuum encapsulation, as shown in figure 6.

Table 1 summarizes the physical dimensions of the support beams. The beam thickness is assumed to be $475 \mu \mathrm{m}$ and the total package thickness and area are $975 \mu \mathrm{m}$ and $12.2 \times 12.2 \mathrm{~mm}^{2}$, respectively.

Figure 6 also depicts the fundamental vibration modes of the lateral and vertical designs as obtained by FEM. FEM simulations demonstrate that the fundamental resonant frequency of the lateral design is approximately $1 \mathrm{kHz}$ while 


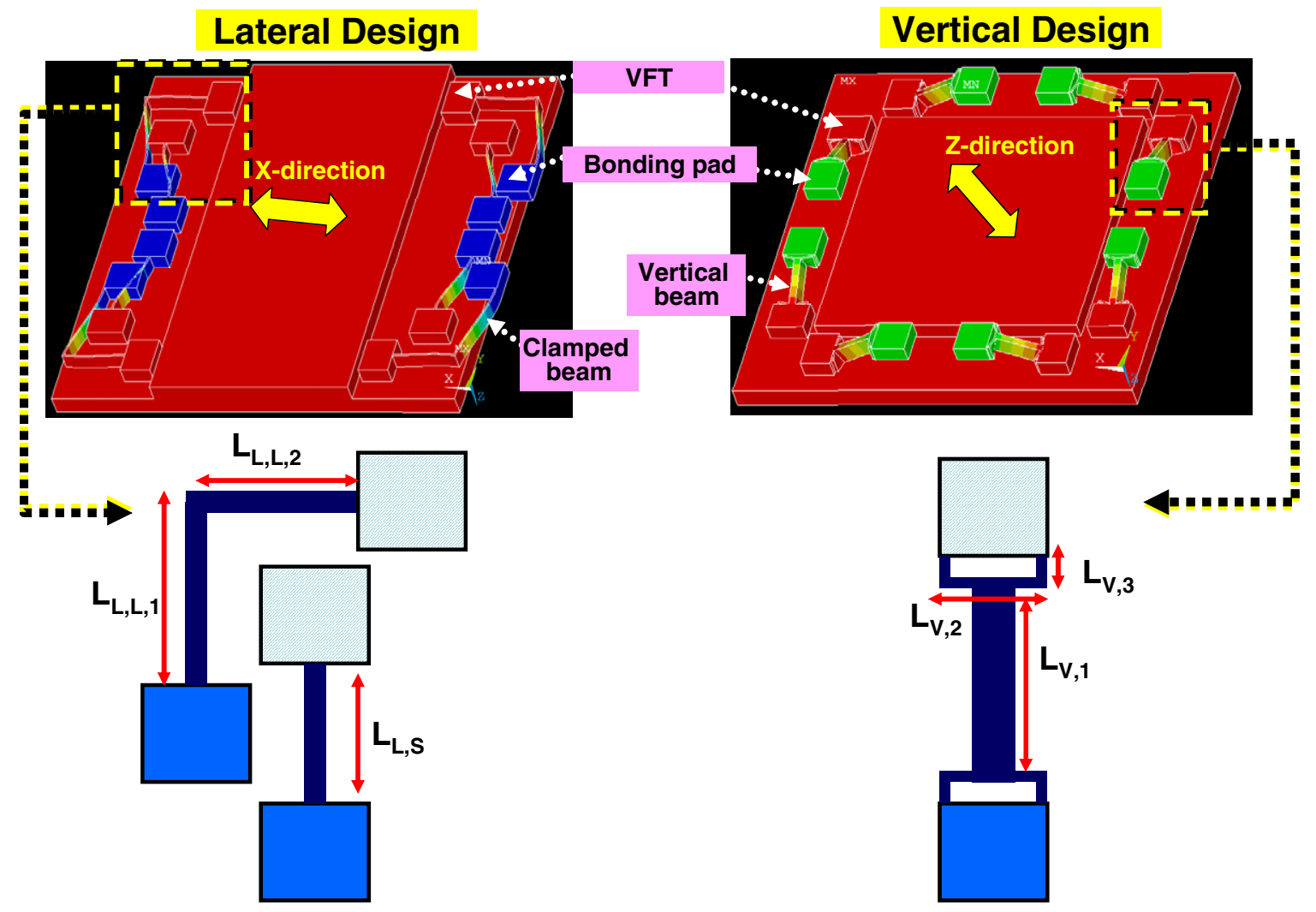

Figure 6. FEM simulation results showing the fundamental resonant modes for lateral (left) and vertical (right) vibration isolator designs. Detailed views and physical dimensions of vibration-isolation beams are also illustrated.

Table 1. Physical dimensions of vibration-isolation beams.

\begin{tabular}{|c|c|c|c|c|c|}
\hline \multicolumn{6}{|c|}{ Lateral design } \\
\hline \multicolumn{2}{|c|}{ Straight beam } & \multicolumn{3}{|c|}{ L-shape beam } & \\
\hline Width & Length $\left(L_{L, S}\right)$ & Width & Length $1\left(L_{L, L, 1}\right)$ & \multicolumn{2}{|l|}{ Length $2\left(\mathrm{~L}_{L, L, 2}\right)$} \\
\hline $50 \mu \mathrm{m}$ & $2 \mathrm{~mm}$ & $50 \mu \mathrm{m}$ & $2.5 \mathrm{~mm}$ & $1.9 \mathrm{~mm}$ & \\
\hline \multicolumn{6}{|c|}{ Vertical design } \\
\hline \multicolumn{2}{|c|}{ Beam 1} & \multicolumn{2}{|r|}{ Beam 2} & \multicolumn{2}{|c|}{ Beam 3} \\
\hline Width & Length $\left(L_{V, 1}\right)$ & Width & Length $\left(L_{V, 2}\right)$ & Width & Length $\left(L_{V, 3}\right.$ \\
\hline $200 \mu \mathrm{m}$ & $1.2 \mathrm{~mm}$ & $50 \mu \mathrm{m}$ & $900 \mu \mathrm{m}$ & $50 \mu \mathrm{m}$ & $50 \mu \mathrm{m}$ \\
\hline
\end{tabular}

that for the vertical design is approximately $4 \mathrm{kHz}$. Thus, both designs have resonant frequencies that are substantially smaller than the resonant frequencies of common MEMS devices such as gyroscopes, and thus, they are expected to realize substantial vibration isolation at these higher frequencies. The designs illustrated in figure 6 incorporate a single vibration-isolation platform. However, designs with multiple cascaded platforms can be readily achieved for higher-order vibration filtering as explained above.

\section{Fabrication}

\subsection{Fabrication process}

The proof-of-concept fabrication process is summarized in figure 7. This process utilizes four wafers: a $4^{\prime \prime}\left(\begin{array}{lll}1 & 0 & 0\end{array}\right)$ $475 \mu \mathrm{m}$ thick double-side polished silicon wafer ( $\mathrm{p}$ type,

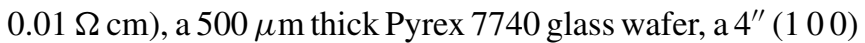
$500 \mu \mathrm{m}$ thick substrate silicon wafer (p type, 10-20 $\Omega \mathrm{cm}$ ), and a guide silicon wafer. This simplified process incorporates only the essential lower part of the package illustrated in figure 5 but doing so readily demonstrates feasibility. Thus, this process incorporates the VFTs through the $475 \mu \mathrm{m}$ thick silicon wafer (in figure 5) but excludes the VFTs through the glass wafer.

On the platform side, a $475 \mu \mathrm{m}$ thick double-side polished silicon wafer is etched to pattern recesses having depths of 2$4 \mu \mathrm{m}$ (figure $7(a)$ ) and anodically bonded in vacuum with a glass wafer to generate the vibration-isolation platform wafer (figure $7(b)$ ). Next, a backside through-wafer deep reactiveion etching (DRIE) is completed to define the VFTs, isolation beams, and bonding pads (figure $7(c)$ ), and a Ti/Au layer is deposited on the backside using e-beam evaporation. The 


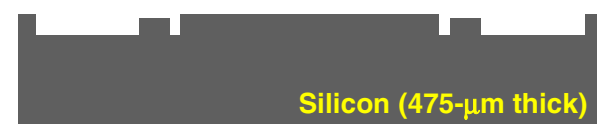

(a)

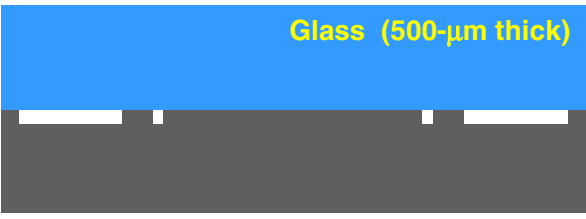

(b)

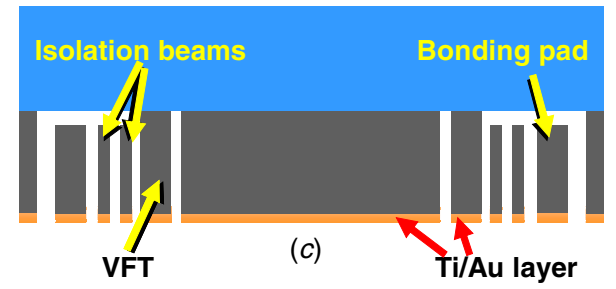

Metal interconnection

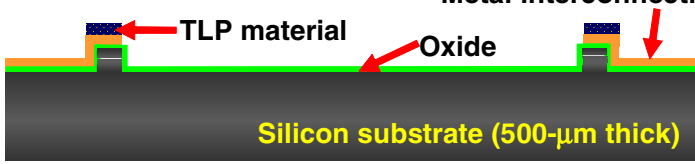

(d)

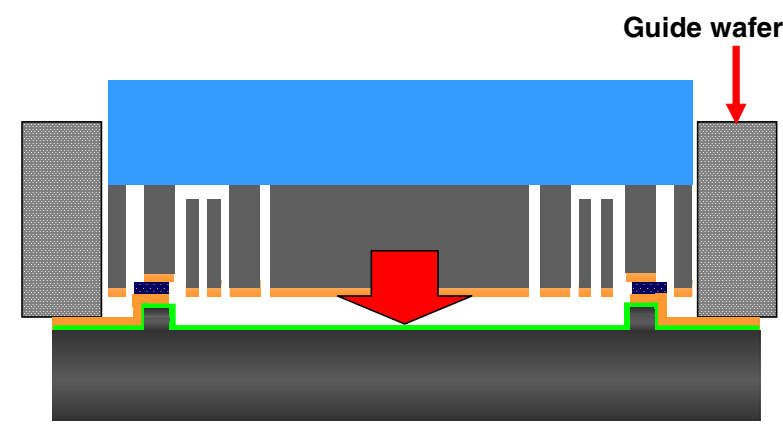

$(e)$

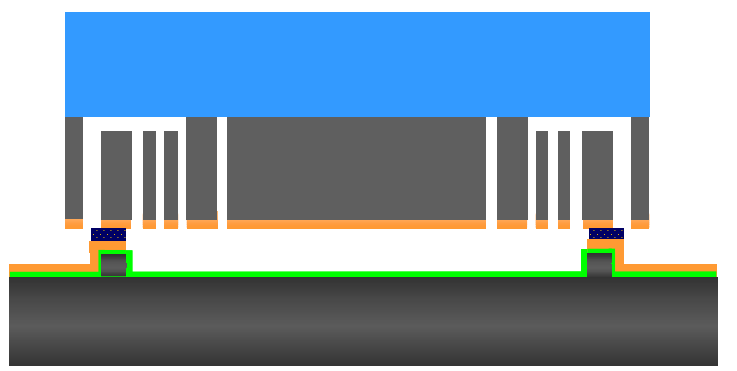

$(f)$

Figure 7. Fabrication process for the vibration-isolation platform. The platform and the substrate are processed separately and bonded together in $(e)$.

metal layer (such as gold) deposited on the beams has two main purposes: to serve as a transient liquid phase (TLP) bonding material (for the bonding pads) $[20,21]$ and to reduce the resistance of the interconnects. Finally, the platform wafer is diced (figure $7(c)$ ).

On the substrate side, DRIE etched islands (heights of 7-8 $\mu \mathrm{m}$ ) are patterned and conformally coated with $1.5 \mu \mathrm{m}$ thick plasma-enhanced chemical vapor deposition (PECVD) oxide for electrical insulation (figure $7(d)$ ). Metal connections are created by depositing and patterning a $\mathrm{Ti} / \mathrm{Au}$ layer on the oxide. Then, a NiCr/In/Au layer is deposited and patterned to form the TLP bonding material (figure $7(d)$ ) $[13,20,21]$. The platform die is bonded to the substrate using TLP bonding through a micromachined guide wafer (figure 7(e)). The guide wafer is removed after bonding and the final structure is shown in figure $7(f)$.

Note that during the TLP bonding, the vibration beams bend until the bonding pads contact the glass wafer while the platform mass is suspended above the substrate. This fabrication step provides sufficient and localized bonding pressure only to the bonding pads. The lateral and vertical springs are designed to limit the maximum bending stresses to values much smaller than the fracture strength of silicon $(0.8-1 \mathrm{GPa})[12,22]$ and then to spring back to their neutral positions after bonding. This fracture protection was verified using FEM simulation and also static loading experiments. The maximum bending stress is also determined by the depth of the recesses shown in figure 7(a), and thus, careful control of the recess depth (2-4 $\mu \mathrm{m}$ in our designs) is required. We conducted FEM simulation to calculate the maximum stress of each beam design developed by a $6 \mu \mathrm{m}$ deflection. The

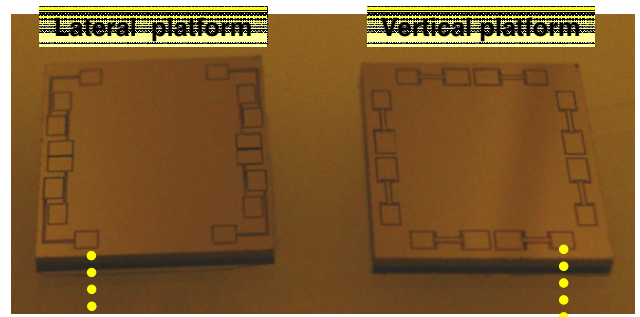

(a)

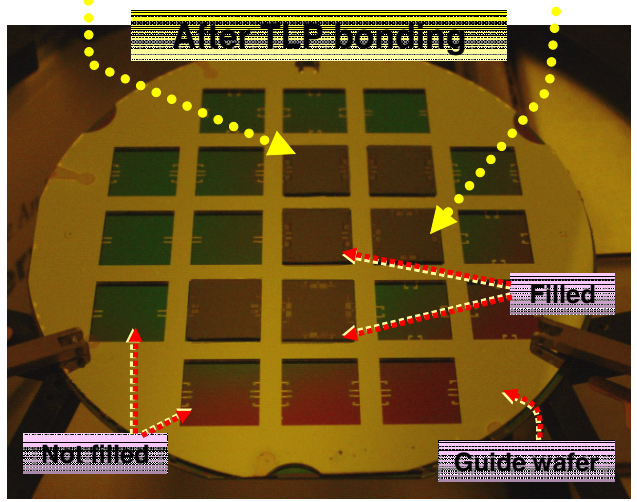

(b)

Figure 8. (a) Fabricated lateral and vertical vibration-isolation platforms. (b) Multiple isolation platforms bonded on a substrate using TLP bonding through a guide wafer.

deflection is slightly larger than our maximum recess depth (4 $\mu \mathrm{m}$ ) to compensate for any fabrication non-uniformity. The maximum stresses of the straight beam and L-shaped beam (of lateral design) are $\sim 2$ and $\sim 7 \mathrm{MPa}$, respectively, and 


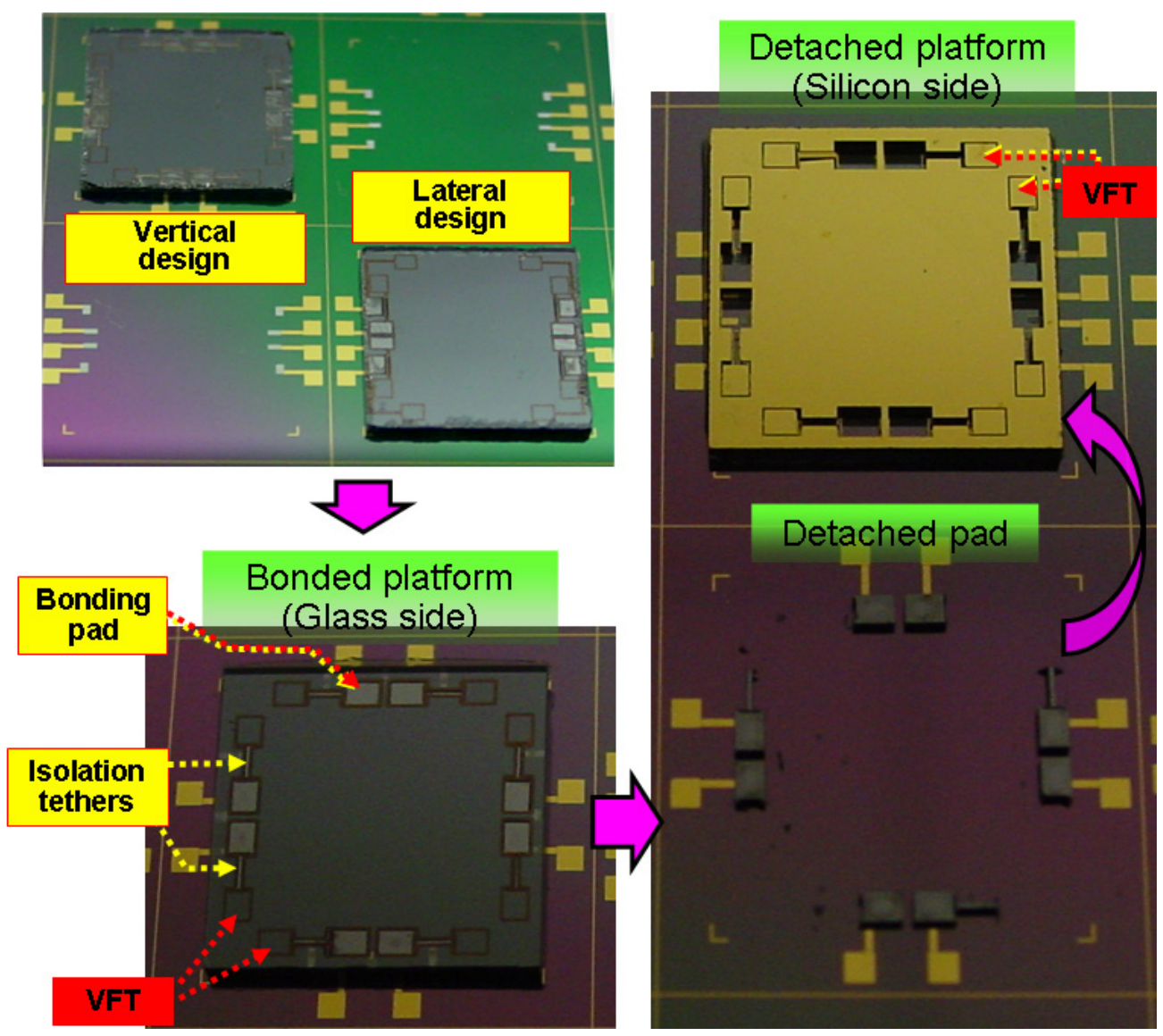

Figure 9. Lateral and vertical vibration-isolation platforms bonded on a single substrate wafer with the vertical platform intentionally detached. The images confirm strong bonding quality.

the maximum stress of the torsion beam (of vertical design) is $\sim 110 \mathrm{MPa}$. All stresses are significantly smaller than the fracture strength of silicon and confirm that no damage occurs during bonding.

Furthermore, the recess depth should be smaller than the height of the DRIE etched islands (shown in figure $7(d)$ ) to secure the suspension of the platform mass during bonding.

\subsection{Fabricated device}

Figure 8 illustrates the fabricated lateral and vertical isolation platforms. Static loading was applied by bending the bonding pad of the platforms in the vertical direction until the pad contacted the glass wafer. No damage was observed, thereby confirming that no damage would result during TLP bonding by excessive bending stress. Figure $8(b)$ illustrates multiple (four) platform dies aligned with a substrate wafer using the guide wafer and simultaneously bonded.

Figure 9 illustrates the bonded platforms following removal of the guide wafer. All platforms were successfully bonded to the single substrate. To investigate bonding quality, the platform was intentionally detached. As shown in figure 9, all bonding pads remained attached to the substrate even after fracture of all vibration beams. Therefore, the TLP bonding achieves uniform and large bonding strength.

\section{Measurements}

The mechanical response of the fabricated vibration-isolation platforms was characterized using a vibration exciter (shaker table, Type 4809 from Brüel \& Kjær) and a laser vibrometer (OFV-3001/OFV 303 from Polytech PI). A schematic of the vibration test setup is depicted in figure 10 . Both the exciter and the laser source are mounted on an anti-vibration table. A test sample (e.g. lateral vibration-isolation platform illustrated in the figure) is mounted on an L-shaped fixture, which is fixed to the head of the exciter, using tacky wax. The exciter is actuated at a frequency $\left(f_{e}\right)$, which is controlled by a custom Labview program and supplementary electronics. The velocity of the excited sample (i.e. lateral platform herein) is measured by the laser vibrometer with feedback to the controller. Finally, the velocity measurement is converted to the displacement of the sample. This sequence is repeated while steadily sweeping the exciter actuation frequency $\left(f_{e}\right)$ over a wide frequency range to generate the frequency response of the test sample. The transmissibility defining vibration isolation is then readily computed.

Figure 11 illustrates the measured result for the lateral isolation platform and compares it with the theoretical calculation derived using equation (1) and the measured resonant frequency and $Q$. Measurements reveal that the platform has a resonant frequency of approximately $1.3 \mathrm{kHz}$ and a $Q$ of approximately 2.2. The measured resonant 


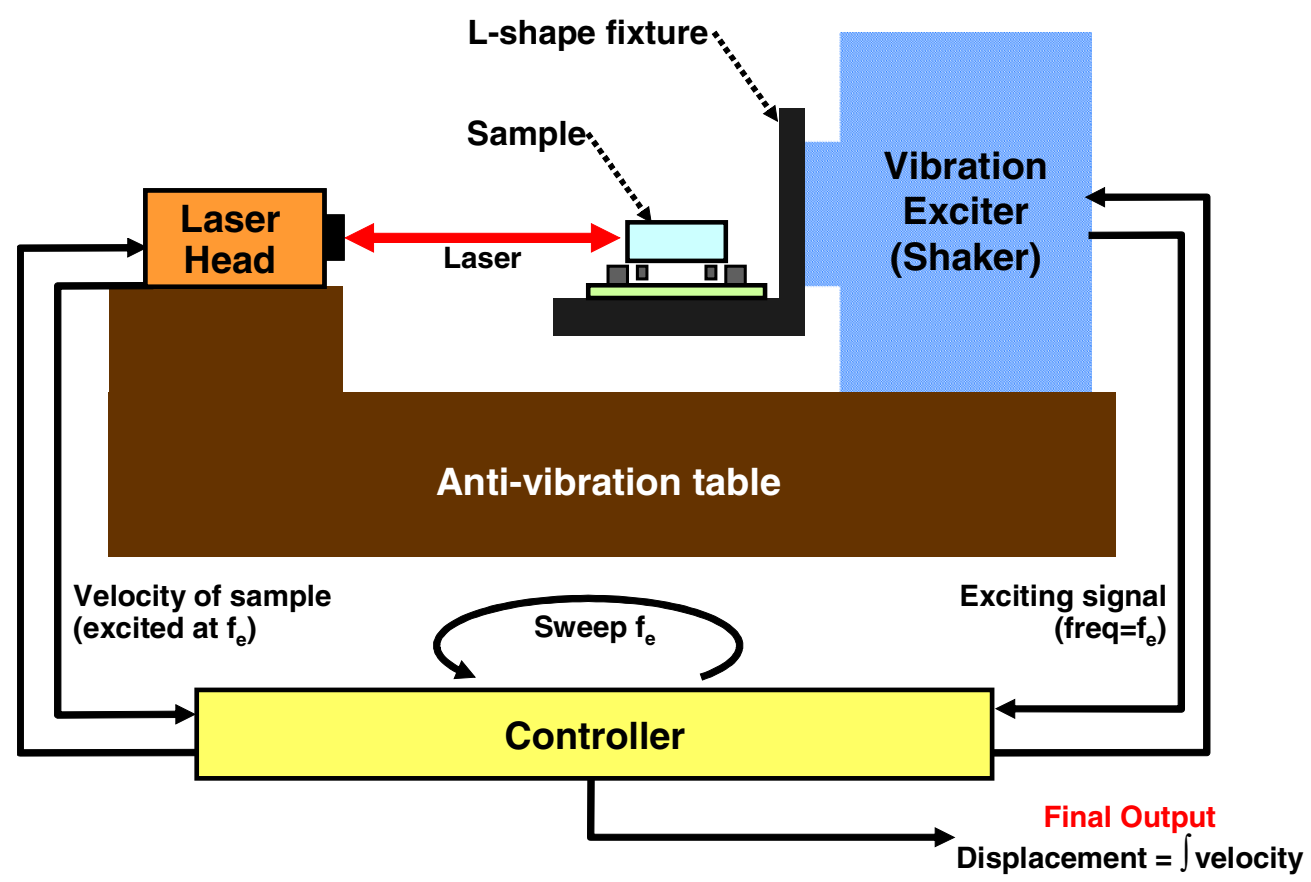

Figure 10. Experimental setup using the vibration exciter and laser vibrometer.

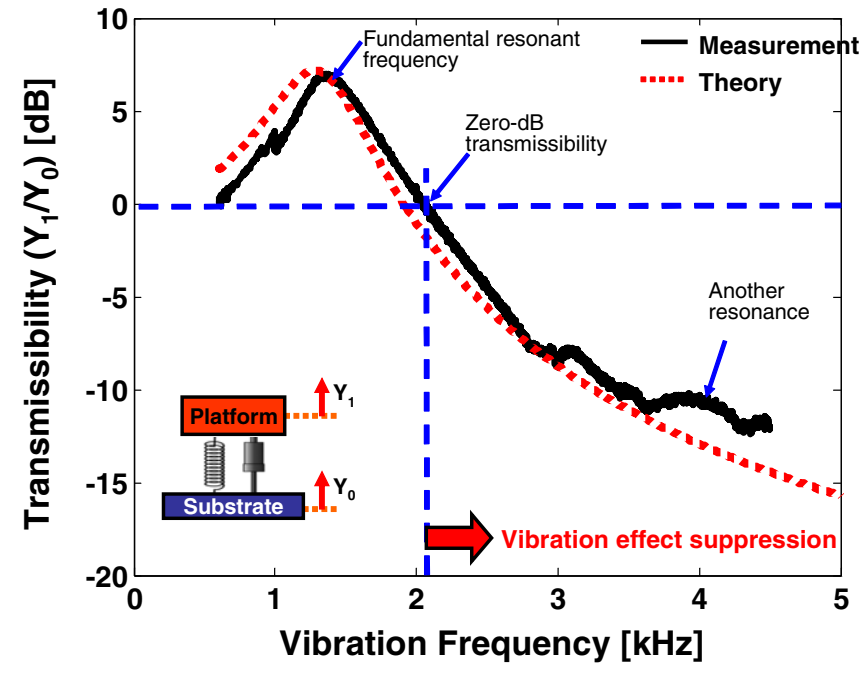

Figure 11. Measured frequency response of the lateral vibration-isolation platform (solid line) and its comparison with theoretical estimation (dotted line). The measurements confirm significant vibration suppression at frequencies exceeding $2.1 \mathrm{kHz}$.

frequency is slightly higher than the resonant frequency predicted by the FEM simulation $(\sim 0.8 \mathrm{kHz})$. The $Q$ remains in the range of 2-3 as reported for MEMS devices [6, 23] that have large silicon mass and resonant frequencies similar to the platform. The transmissibility $\left(Y_{1} / Y_{0}\right)$ is derived by dividing the frequency response of the platform mass $\left(Y_{1}\right)$ by that of the platform substrate $\left(Y_{0}\right)$. The results demonstrate that vibration isolation is achieved at vibrations whose frequencies exceed $2.1 \mathrm{kHz}$ because the transmissibility is less than 1 beyond that frequency (i.e. $0 \mathrm{~dB}$ in figure 11).
The results of figure 11 also demonstrate good agreement between measurements and the theory. However, the measured transmissibility exceeds the calculated values at input frequencies above approximately $3.7 \mathrm{kHz}$. This arises from the influence of a second mode at approximately $4 \mathrm{kHz}$, and the FEM simulation confirms that a torsion mode exists at approximately $3.8 \mathrm{kHz}$. The attenuation at the resonant frequency of the example MEMS gyroscopes will therefore be larger than the simulated values predicted in figure 3 .

The electrical resistance between the VTF and the bonding pads is measured using a digital multimeter and ranges from 4 to $11 \Omega$ depending on the spring design. Because of the physical dimensions of vibration beams, the lateral designs have greater resistance than the vertical designs.

\section{Conclusions}

This paper presents analyses and preliminary results for integrating multiple vibration-isolation platforms with resonating MEMS devices using wafer-level microfabrication. The isolation platform functions as a mechanical LPF and consists of platform mass, vibration-isolation beams, VFTs, and bonding pads. It is also possible to implement highorder filters and achieve larger vibration-effect isolation simply by cascading multiple isolation platforms via layout-level modification. Both lateral and vertical designs are fabricated by a single fabrication process, and therefore, this concept can also realize a multi-dimensional LPF. The platform mass is designed to be significantly larger than the device mass to decrease the resonant frequency of the platform and to minimize any negative side-effects of integrating the devices. The vibration beams function as both mechanical springs 
and electrical interconnects. Different beam designs are employed for lateral versus vertical vibration isolation. The lateral isolator design uses clamped-clamped beams and the vertical isolator design uses torsion beams. Each design has a fundamental resonant frequency (lateral: $\sim 1 \mathrm{kHz}$, vertical: $\sim 4 \mathrm{kHz}$ ) substantially smaller than that of common resonant MEMS devices including gyroscopes.

A proof-of-concept design was fabricated and test results are presented herein. The isolator beams were fabricated using $475 \mu \mathrm{m}$ thick silicon and the package has a net thickness of $975 \mu \mathrm{m}$ and area of $12.2 \times 12.2 \mathrm{~mm}^{2}$. Both the lateral and vertical platforms were mounted on a silicon substrate and bonded using TLP bonding. The result leads to a complete, $3 \mathrm{D}$ vibration isolation system created via a single fabrication process. The platform has contact resistance ranging from 4 to $11 \Omega$ depending on the beam design. Measurements using a laser-Doppler vibrometer confirm that external vibration with frequencies exceeding $2.1 \mathrm{kHz}$ are substantially isolated in the lateral vibration-isolation platform.

This design concept is compatible and easily integrated with a previously reported environment-resistant package. The result exploits the combined functionalities to yield a vacuum package with thermal and vibration isolation without major design changes or additional resources.

\section{Acknowledgments}

This work was supported by DARPA's HERMIT program (contract no W31P4Q-04-1-R001). The authors especially thank Professor K Grosh and Dr R Littrell (Mechanical Engineering, University of Michigan) for their help in conducting the laser vibrometer measurements.

\section{References}

[1] Schofield A R, Trusov A A and Shkel A M 2007 Multi-degree of freedom tuning fork gyroscope demonstrating shock rejection Proc. IEEE Sensors pp 120-3

[2] Geen J A, Sherman S J, Chang J F and Lewis S R 2002 Single-chip surface micromachined integrated gyroscope with 500/h Allan deviation IEEE J. Solid-State Circuits 37 1860-6

[3] Yoon S W, Lee S W, Perkins N C and Najafi K 2007 Vibration sensitivity of MEMS tuning fork gyroscopes Proc. IEEE Sensors Conf. (Atlanta, GA, USA) pp 115-9

[4] Balandin D V, Bolotnik N N and Pilkey W D 2001 Optimal Protection from Impact, Shock and Vibration (Amsterdam: Gordon \& Breach)

[5] Braman T and Grossman O 2006 Designing vibration and shock isolation systems for micro electrical machined based inertial measurement units Proc. IEEE/ION Position, Location, and Navigation Symposium (PLANS) pp 400-4
[6] Reid J R, Bright V M and Kosinski J A 1998 A micromachined vibration isolation system for reducing the vibration sensitivity of surface transverse wave resonators IEEE Trans. Ultrason. Ferroelectr. Freq. Control 45 528-34

[7] Dean R et al 2002 Vibration isolation of MEMS sensors for aerospace applications Proc. IMAPS Int. Conf. and Exhibition on Advanced Packaging and Systems (Reno, NV, USA) pp 166-70

[8] Dean R, Flowers G, Sanders N, Horvath R, Kranz M and Whitley M 2005 Micromachined vibration isolation filters to enhance packaging for mechanically harsh environments J. Microelectron. Electron. Packag. $2223-31$

[9] Kim S J, Dean R, Flowers G and Chen C 2009 Active vibration control and isolation for micromachined devices ASME J. Mech. Des. 131091002

[10] Meyer Y, Verdot T, Collet M, Baborowski J and Muralt P 2007 Active isolation of electronic micro-components with piezoelectrically transduced silicon MEMS devices Smart Mater. Struct. 16 128-34

[11] Inman D J 2000 Engineering Vibration 2nd edn (Englewood Cliffs, NJ: Prentice-Hall)

[12] Yoon S W 2009 Vibration isolation and shock protection for MEMS PhD Dissertation University of Michigan

[13] Lee S H, Lee S W and Najafi K 2007 A generic environment-resistant packaging technology for MEMS Proc. Int. Solid-State Sensors, Actuators and Microsystems Conf. (TRANSDUCERS) (Lyon, France) pp 335-8

[14] Meirovitch L 1986 Elements of Vibration Analysis (New York: McGraw-Hill)

[15] Zaman M F, Sharma A and Ayazi F 2006 High performance matched-mode tuning fork gyroscope Proc. 19th IEEE Int. Conf. on Micro Electro Mechanical Systems (Istanbul, Turkey) pp 66-9

[16] Song I and Lee B 2004 MEMS-based angular rate sensors Proc. IEEE Sensors vol 2 pp 650-3

[17] An S, Oh Y S, Park K Y, Lee S S and Song C M 1999 Dual-axis microgyroscope with closed-loop detection Sensors Actuators A 73 1-6

[18] Lee S, Cho J and Najafi K 2007 Fabrication of vertical comb electrodes using selective anodic bonding Proc. IEEE Int. Conf. on Micro Electro Mechanical Systems (MEMS) (Kobe, Japan) pp 349-52

[19] Kim J, Park S, Kwak D, Ko H and Cho D D 2005 An $x$-axis single-crystalline silicon microgyroscope fabricated by the extended SBM process J. Microelectromech. Syst. 14 444-55

[20] Welch W C and Najafi K 2008 Gold-indium transient liquid phase (TLP) wafer bonding for MEMS vacuum packaging Proc. IEEE Int. Conf. on Micro Electro Mechanical Systems (MEMS) (Tucson, AZ, USA) pp 806-9

[21] Welch W C and Najafi K 2007 Nickel-tin transient liquid phase (TLP) wafer bonding for MEMS vacuum packaging Proc. Int. Solid-State Sensors, Actuators and Microsystems Conf. (TRANSDUCERS) (Lyon, France) pp 1327-8

[22] Yee J K, Yang H H and Judy J W 2003 Shock resistance of ferromagnetic micromechanical magnetometers Sensors Actuators A 103 242-52

[23] Abdolvand R, Amini B V and Ayazi F 2007 Sub-micro-gravity in-plane accelerometers with reduced capacitive gaps and extra seismic mass J. Microelectromech. Syst. 16 1036-43 